\title{
Continuity of vortices from the hadronic to the color-flavor locked phase in dense matter
}

\author{
Mark G. Alford, ${ }^{1}$ Gordon Baym, ${ }^{2,3}$ Kenji Fukushima, ${ }^{4}$ Tetsuo Hatsuda,,${ }^{3,5}$ and Motoi Tachibana ${ }^{6}$ \\ ${ }^{1}$ Department of Physics, Washington University, St. Louis, Missouri 63130, USA \\ ${ }^{2}$ Department of Physics, University of Illinois, 1110 West Green Street, Urbana, Illinois 61801-3080, USA \\ ${ }^{3}$ iTHES Research Group and iTHEMS Program, RIKEN, Wako, Saitama 351-0198, Japan \\ ${ }^{4}$ Department of Physics, The University of Tokyo, 7-3-1 Hongo, Bunkyo-ku, Tokyo 113-0033, Japan \\ ${ }^{5}$ Nishina Center, RIKEN, Wako, Saitama 351-0198, Japan \\ ${ }^{6}$ Department of Physics, Saga University, Saga 840-8502, Japan
}

(Received 21 March 2018; published 7 February 2019)

\begin{abstract}
We study how vortices in dense superfluid hadronic matter can connect to vortices in superfluid quark matter, as in rotating neutron stars, focusing on the extent to which quark-hadron continuity can be maintained. As we show, a singly quantized vortex in three-flavor symmetric hadronic matter can connect smoothly to a singly quantized non-Abelian vortex in three-flavor symmetric quark matter in the colorflavor locked phase, without the necessity for boojums appearing at the transition.
\end{abstract}

DOI: 10.1103/PhysRevD.99.036004

\section{INTRODUCTION}

In a rotating neutron star, the superfluid componentsthe nuclear liquid at lower densities and a possible colorflavor locked (CFL) quark phase [1] at higher densities in the interior-carry angular momentum in the form of quantized vortices. How, we ask, are the vortices in these two phases connected? Can one have continuity or must there be a discontinuity? How do the possible connections depend on the particular flavor structure of the matter? In the ground state of dense matter, the picture of quarkhadron continuity [2,3] is that as the baryon density is increased matter undergoes a smooth crossover from the hadronic phase to the quark phase. By studying how such vortices connect we can shed further light on whether the notion of quark-hadron continuity can be extended to angular momentum carrying states of dense hadronic matter.

To summarize the problem in matching hadronic with CFL vortices we note that superfluid vortices in the Bardeen-Cooper-Schrieffer (BCS)-paired hadronic phase have quantized circulation, $C_{\mathrm{B}}$, i.e.,

$$
C_{\mathrm{B}}=\oint_{\mathcal{C}} \vec{v} \cdot d \vec{\ell}=2 \pi \frac{\nu_{\mathrm{B}}}{2 \mu_{\mathrm{B}}},
$$

where the contour $\mathcal{C}$ of integration encircles the vortex, $\mu_{\mathrm{B}}$ is the baryon chemical potential, and $\nu_{\mathrm{B}}$ is an integer. We

Published by the American Physical Society under the terms of the Creative Commons Attribution 4.0 International license. Further distribution of this work must maintain attribution to the author(s) and the published article's title, journal citation, and DOI. Funded by SCOAP. detail this result further below. (We work in units $\hbar=$ $c=1$.) All but singly quantized vortices $\left(\nu_{\mathrm{B}}= \pm 1\right)$ are unstable. In a BCS-paired CFL quark phase on the other hand, the simple Abelian vortex [4,5], the analog of the hadronic vortex, has circulation [6]

$$
C_{\mathrm{A}}=\oint_{\mathcal{C}} \vec{v} \cdot d \vec{\ell}=2 \pi \frac{\nu_{\mathrm{A}}}{2 \mu_{\mathrm{q}}},
$$

where $\mu_{\mathrm{q}}=\mu_{\mathrm{B}} / 3$ is the quark chemical potential, and again $\nu_{\mathrm{A}}$ is an integer. Singly quantized $U(1)_{\mathrm{B}}$ Abelian vortices in the quark phase have three times the circulation of singly quantized hadronic vortices.

Thus if one were to imagine a singly quantized hadronic vortex turning into a singly quantized Abelian CFL vortex, the baryon velocity would have to jump discontinuously by a factor of 3 from the hadronic to the quark phase, eliminating any possibility of quark-hadron continuity. Indeed, to make the velocity continuous one would have to join three hadronic vortices to a single Abelian quark vortex, as illustrated in Fig. 1(a). Such a join is known as a boojum [7].

Single Abelian vortices in the CFL phase, however, are unstable against separating into three non-Abelian vortices [8-10], each of which has $1 / 3$ the circulation of the Abelian vortex. ${ }^{1}$ Thus one might envisage a join with a continuous

\footnotetext{
${ }^{1}$ In Ref. [8] these configurations were referred to as "semisuperfluid strings"; however, we call them "non-Abelian vortices" to emphasize the presence of non-Abelian color magnetic flux in the core combined with vortexlike global rotation of the quark condensate.
} 


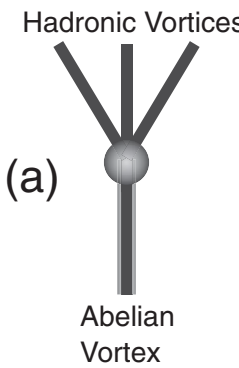

(b)

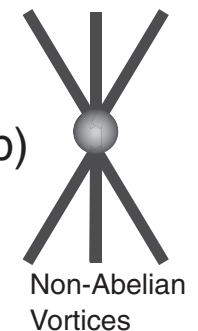

(c)

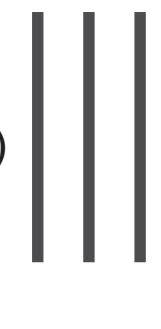

FIG. 1. Schematic illustrations for connecting vortices: (a) If angular momentum in the CFL phase is carried by Abelian CFL vortices then in the crossover to the hadronic phase a boojum (shaded circle) joins three hadronic vortices to a single Abelian CFL vortex; (b) because Abelian CFL vortices are unstable, three hadronic vortices match onto three non-Abelian CFL vortices through a modified boojum; or (c) each hadronic vortex matches onto a single non-Abelian CFL vortex without the need for a boojum.

baryon velocity, as shown in Fig. 1(b), where a boojum connects three hadronic vortices with three non-Abelian CFL vortices $[11,12]$. However, as we discuss in this paper, one does not have to make a join involving three vortices in the hadronic phase, but rather one can make a baryonvelocity conserving join between a single hadronic vortex and a single non-Abelian vortex in the CFL phase, as shown in Fig. 1(c), without any need for a boojum. To the extent that the various flavor quantum numbers permit a smooth transition from the hadronic to the CFL quark phase, angular momentum carrying states remain consistent with quark-hadron continuity.

To spell out this picture in detail, we first discuss more precisely the nature of quark-hadron continuity between the hadronic and quark phases. On the deconfined quark side the (ideal) CFL phase contains $u$ (up), $d$ (down), and $s$ (strange) quarks, all with the same mass, with a Fermi sea equally populated with all three flavors and all three colors of quarks. The corresponding hadronic phase, three-flavor hyperonic matter, contains all members of the light baryon flavor octet, $n, p, \Lambda, \Sigma^{0}, \Sigma^{ \pm}, \Xi^{0}$, and $\Xi^{-}$, all of the same mass. In the ground state at finite density, the particles populate a Fermi sea with all states of the octet equally present.

Both phases break chiral symmetry [1] and $U(1)_{\mathrm{B}}$, with the same symmetry breaking pattern $\left[S U(3)_{\mathrm{L}} \otimes\right.$ $\left.S U(3)_{\mathrm{R}} \otimes U(1)_{\mathrm{B}} \rightarrow S U(3)_{\mathrm{V}}\right]$. In the hadronic phase, the dibaryon condensate, which breaks $U(1)_{\mathrm{B}}$, is formed from two paired flavor octets, while in the CFL phase, a diquark condensate is formed, which in the unitary gauge has the same color-flavor orientation everywhere. ${ }^{2}$ Also, in the hadronic phase, chiral symmetry is spontaneously broken by a quark-antiquark chiral condensate, producing a light octet of pseudoscalar mesons, i.e., $\pi^{0}, \pi^{ \pm}, K^{0}, \bar{K}^{0}, K^{ \pm}$, and

\footnotetext{
${ }^{2}$ With full three-flavor symmetry, CFL pairing is the most stable $[13,14]$.
}

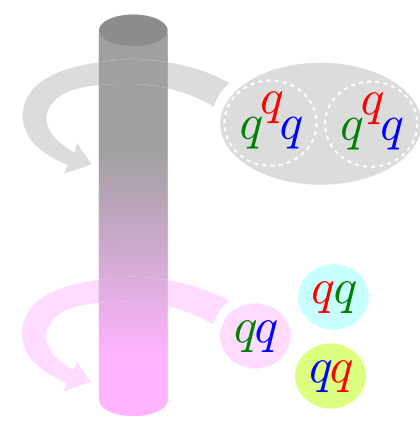

FIG. 2. Schematic illustration of the smooth evolution of a hadronic vortex into a non-Abelian CFL vortex. In the hadronic phase, the phase of the condensate corresponding to paired baryons (six quarks) increases by $2 \pi$ in winding around the vortex core. In the CFL phase in the gauge-fixed picture, one component of the order parameter picks up a phase $2 \pi$ in winding, as shown. In the gauge-invariant picture the phase of the entire six-quark order parameter changes by $2 \pi$ in winding.

$\eta$, while in the CFL phase, the diquark condensate spontaneously breaks chiral symmetry, producing a light octet of pseudoscalar mesons [15-17]. Previous studies $[2,3,18,19]$ have established the continuity between the low-energy excitations of such three-flavor hadronic and three-flavor quark matter. ${ }^{3}$ The nine single-quark excitations of different colors and flavors can be mapped, in the unitary gauge, onto the baryon octet plus a baryon singlet, which is usually not mentioned in discussions of the confined phase because it is much heavier than the octet baryons [3].

One can further understand quark-hadron continuity in terms of the anomaly-induced coupling between the chiral and diquark condensates [21,22]. The implications of quark-hadron continuity for the QCD phase diagram are reviewed in Ref. [23], and for neutron stars in Ref. [24].

Figure 2 summarizes our results. In the confined phase (upper half of the figure) the hadronic vortex carries angular momentum via the circulation of a gauge-invariant dibaryon condensate that acquires a phase of $2 \pi$ when transported around the core. This vortex can be continuously connected to a non-Abelian CFL vortex [8] in the CFL quark phase (lower half of the figure) where the vortex has the same baryon circulation, but it arises in the unitary gauge from three diquark condensates, one of which acquires a phase of $2 \pi$ when transported around the core. On the other hand, in the gauge-invariant picture, described in detail in Sec. III D, the phase increase is attributed to the entire six-quark order parameter.

This paper is organized as follows. In Sec. II we review the generic properties of vortices in a superfluid. In Sec. III we discuss the vortex configurations that exist in

\footnotetext{
${ }^{3}$ This continuity is an example of the complementarity between the confined and Higgs phases of a non-Abelian gauge theory [20].
} 
three-flavor hadronic and quark matter. After discussions of hadronic vortices in Sec. III A, we describe two different vortex configurations that have been constructed in threeflavor quark matter, Abelian CFL vortices in Sec. III B and non-Abelian CFL vortices in Sec. III C, and then we show how a non-Abelian vortex can be continuously connected with a hadronic vortex. In Sec. III D we show how these non-Abelian vortices can be understood in a gauge-invariant description, focusing in Sec. III D 2 on the continuity of flavored vortices. Finally, in Sec. IV we discuss the role of color magnetic flux. We focus throughout on the properties of connecting single vortices, and leave the discussion of an array of vortices in the CFL phase at finite rotation for the future.

\section{VORTEX QUANTIZATION AND CIRCULATION}

We first review the basics of vortex quantization, circulation, and angular momentum, which are common to all the vortices we discuss here: hadronic vortices, Abelian CFL vortices, and CFL vortices carrying nonAbelian color flux.

Quantized vortices arise in superfluids under rotation. A superfluid can be described by a complex scalar field; the ground state expectation value $\Phi(\vec{r}, t)$ of the field, in the conventional description in terms of broken symmetry, represents the condensate of bosons (or Cooper pairs of fermions) that gives rise to superfluidity. The Hamiltonian for the field is invariant under a global $U(1)$ symmetry, so that the number of bosons or fermions is conserved by the dynamics. However, if $\Phi$ is nonzero then the ground state of the Hamiltonian spontaneously breaks the $U(1)$ symmetry.

In general, the condensate can be written in terms of its modulus and phase $\phi$ as

$$
\Phi=e^{i \phi}|\Phi| .
$$

In the local rest frame of the condensate,

$$
\phi=-\mu_{\mathrm{s}} t
$$

where $\mu_{\mathrm{s}}$ is the chemical potential of the conserved particles in the ground state, namely the minimum energy required to add one boson or one pair of fermions to the system. Boosting to a frame in which the condensate is in uniform motion [25], we find

$$
\phi=p_{\nu} x^{\nu}=\vec{p} \cdot \vec{r}-\mu t,
$$

where $\quad p_{\nu} p^{\nu}=-\mu_{s}^{2} \quad$ and $\quad \mu=\gamma(v) \mu_{s} \quad$ with $\quad \gamma(v) \equiv$ $1 / \sqrt{1-v^{2}}$. The superfluid velocity is simply

$$
\vec{v}=\frac{\vec{p}}{\left|p_{0}\right|}=\frac{\vec{p}}{\mu}
$$

We can thus write the momentum carried by the unit of conserved charge and the chemical potential as

$$
\vec{p}=\vec{\nabla} \phi(\vec{r}, t), \quad \mu=-\frac{\partial \phi(\vec{r}, t)}{\partial t}
$$

for general space-time dependent $\phi$.

For a static superfluid vortex, $\phi(\vec{r}, t)=\phi(\vec{r})-\mu t$; thus

$$
\Phi(\vec{r})=e^{i \phi(\vec{r})-i \mu t}|\Phi(\vec{r})|,
$$

where $|\Phi(\vec{r})|$ is vanishing at the center of the vortex and in uniform density matter is independent of position well outside the vortex core. Far from the vortex core the only spatial variation is in the phase $\phi(\vec{r})$.

For the mathematically simplest vortex aligned along the $z$ axis, $\phi=\nu \varphi$, where $\varphi$ is the azimuthal angle. Thus the momentum per particle or pair is

$$
\vec{p}(r)=\vec{\nabla} \phi=\frac{\nu}{r} \hat{\varphi}
$$

where $r$ is the distance from the vortex core and $\hat{\varphi}$ is a unit vector in the $\varphi$ direction. From Eq. (6) the superfluid velocity is

$$
v(r)=\frac{\nu}{\mu r} \hat{\varphi} .
$$

Integrating $\vec{p}$ along a closed contour $\mathcal{C}$ surrounding the vortex we obtain the total change $\Delta \phi$ in the phase,

$$
\Delta \phi=\oint_{\mathcal{C}} \vec{p} \cdot d \vec{\ell}=2 \pi \nu
$$

In a three-dimensional system, the winding number $\nu$ must be an integer. From Eqs. (6) and (11) [or from Eq. (10)] the superfluid velocity obeys the circulation condition,

$$
C=\oint_{\mathcal{C}} \vec{v} \cdot d \vec{\ell}=2 \pi \frac{\nu}{\mu}
$$

as mentioned in the introduction.

Lastly we compute the angular momentum, $L_{z}$, of a vortex centered on the $z$ axis. From Eq. (7) the local azimuthal momentum density is $p_{\varphi} n$ where $n$ is the particle density (as distinguished from the condensate density), which is independent of $\varphi$. Thus

$$
L_{z}=\int d^{3} r r p_{\varphi} n(r)=\nu \int 2 \pi r d r d z n(r)=N \nu,
$$

where $N$ is the total number of particles or pairs. The angular momentum per particle for bosons or per fermion pair is simply $\nu$, the winding number of the vortex. 


\section{VORTICES IN HADRONIC AND CFL QUARK MATTER}

We now consider the circulation and the angular momentum associated with vortices in hadronic and CFL quark matter.

\section{A. Hadronic vortices}

In the $S U(3)$ classification, baryon pairs can be decomposed into irreducible representations as

$$
\mathbf{8} \otimes \mathbf{8}=\underbrace{\mathbf{1} \oplus \mathbf{8} \oplus \mathbf{2 7}}_{\text {sym }} \oplus \underbrace{\mathbf{8} \oplus \mathbf{1 0} \oplus \mathbf{1 0}^{*}}_{\text {anti-sym }} .
$$

Here and below, sym and anti-sym stand for the symmetry under the flavor exchange of two baryons. The baryonbaryon interaction in the $S U(3)$ limit is most attractive in the flavor-singlet channel (1 representation) [26] with a pairing gap of the form $\Delta_{\mathrm{B}}^{(\mathbf{1})}=\left\langle-\sqrt{\frac{1}{8}}[\Lambda \Lambda]_{\mathrm{sym}}+\sqrt{\frac{3}{8}}[\Sigma \Sigma]_{\mathrm{sym}}+\right.$ $\left.\sqrt{\frac{4}{8}}[N \Xi]_{\text {sym }}\right\rangle$. In the ground state of three-flavor hyperonic matter, flavor nonsinglet pairings in other attractive channels can coexist with the flavor-singlet pairing, e.g., the standard nucleon pairing in the spin-singlet isospin-triplet channel, $\Delta_{\mathrm{B}}^{(27)}=\left\langle[N N]_{\mathrm{sym}}\right\rangle$, and the possible pairing in the spin-singlet isospin-doublet channel, $\Delta_{\mathrm{B}}^{\left(\boldsymbol{8}_{\mathrm{sym}}\right)}=\left\langle-\frac{1}{10}[N \Lambda]_{\text {sym }}+\sqrt{\frac{9}{10}}[N \Sigma]_{\text {sym }}\right\rangle[27]$.

In any of these pairings, the chemical potential entering Eq. (12) is $2 \mu_{\mathrm{B}}$, that of a pair of baryons. Therefore, no matter whether it is flavor singlet or nonsinglet, a hadronic vortex with winding number $\nu_{\mathrm{B}}$ has circulation $2 \pi \nu_{\mathrm{B}} /\left(2 \mu_{\mathrm{B}}\right)$, Eq. (1). The corresponding angular momentum per baryon is [see Eq. (13)]

$$
\frac{L_{\mathrm{B} z}}{N_{\mathrm{B}}}=\frac{1}{2} \nu_{\mathrm{B}},
$$

since there are $N_{\mathrm{B}} / 2$ pairs in the system.

\section{B. Abelian CFL vortices}

The order parameter of quark matter in the CFL phase in the unitary gauge can be written in terms of the color and flavor triplet diquark operator [1]

$$
\hat{\Phi}^{\alpha i}=\mathcal{N} \epsilon^{\alpha \beta \gamma} \epsilon^{i j k} q_{\beta j} C \gamma_{5} q_{\gamma k},
$$

where $C=i \gamma_{0} \gamma_{2}$ is the charge conjugation operator, and greek and latin letters denote color and flavor indices, respectively; $\mathcal{N}$ is a normalization constant. The order parameter is then

$$
\Phi^{\alpha i}=\left\langle\hat{\Phi}^{\alpha i}\right\rangle .
$$

The matrix $\Phi^{\alpha i}$ can be diagonalized by a combination of color and flavor rotations, so that without loss of generality we write

$$
\Phi=\left(\begin{array}{ccc}
\Phi^{\bar{r} \bar{u}} & 0 & 0 \\
0 & \Phi^{\bar{g}} \bar{d} & 0 \\
0 & 0 & \Phi^{\bar{b} \bar{s}}
\end{array}\right)
$$

where $r, g, b(\bar{r}, \bar{g}, \bar{b})$ denote colors (anticolors) and $u, d, s$ flavors; in the ground state, $\Phi^{\bar{r} \bar{u}}=\Phi^{\bar{g} \bar{d}}=\Phi^{\bar{b} \bar{s}}=\Delta_{\mathrm{CFL}}$.

Naively one would expect the angular momentum carrying states with lowest energy per unit of angular momentum to be global $U(1)_{\mathrm{B}}$ or "Abelian CFL" vortices. In these vortices each of the three nonzero components of the order parameter winds around the core of the vortex, so for an Abelian CFL vortex aligned along the $z$ axis the order parameter assumes the form

$$
\Phi^{\mathrm{A}}=\Delta_{\mathrm{CFL}} e^{i \nu_{\mathrm{A}} \varphi}\left(\begin{array}{ccc}
f(r) & 0 & 0 \\
0 & f(r) & 0 \\
0 & 0 & f(r)
\end{array}\right),
$$

where $f(r)$ varies monotonically from 0 at $r=0$ to unity as $r \rightarrow \infty$, with $\nu_{\mathrm{A}}$ being the winding number of the Abelian CFL vortex.

The quark chemical potential is $\mu_{\mathrm{q}}=\mu_{\mathrm{B}} / 3$, and thus the chemical potential per quark pair is $2 \mu_{\mathrm{q}}=\frac{2}{3} \mu_{\mathrm{B}}$, so from Eqs. (6) and (7) and the total momentum per quark pair in the condensate is

$$
\vec{p}=\frac{2}{3} \mu_{\mathrm{B}} \vec{v},
$$

where as before $\vec{v}$ is the superfluid velocity, so the circulation is

$$
C^{\mathrm{A}}=\frac{3 \nu_{\mathrm{A}}}{2 \mu_{\mathrm{B}}} \oint d \vec{\ell} \cdot \vec{\nabla} \varphi=\frac{3 \nu_{\mathrm{A}}}{2} \cdot \frac{2 \pi}{\mu_{\mathrm{B}}} .
$$

The angular momentum per baryon of the vortex is

$$
\frac{L_{\mathrm{A} z}}{N_{\mathrm{B}}}=\frac{3}{2} \nu_{\mathrm{A}} .
$$

We now ask how the vortices in hadronic matter would match on to Abelian vortices in CFL quark matter at a crossover between these phases. If the superfluid velocity, and hence the circulation, Eq. (12), and angular momentum per baryon, Eq. (13), do not match in the two phases, then quark-hadron continuity would be violated. By comparing Eqs. (1) and (21), or equivalently (15) and (22), we see that matching would require

$$
\nu_{\mathrm{B}}=3 \nu_{\mathrm{A}} .
$$


The matching relation (23) implies that three singly quantized hadronic vortices should merge into one Abelian CFL vortex, violating quark-hadron continuity in states with finite angular momentum. This merging would require a boojum [7] at the interface between the two phases, as sketched in Fig. 1(a). As we discuss in the next section, the violation need not be present for the more stable non-Abelian vortices in the CFL phase.

\section{Non-Abelian CFL vortices}

An Abelian CFL vortex is energetically unstable against formation of three non-Abelian vortices [8,9]. The condensate of the antired-antiup $(\bar{r} \bar{u})$ non-Abelian vortex is

$$
\Phi^{(1)}=\Delta_{\mathrm{CFL}}\left(\begin{array}{ccc}
e^{i \nu_{1} \varphi} f(r) & 0 & 0 \\
0 & g(r) & 0 \\
0 & 0 & g(r)
\end{array}\right),
$$

with corresponding gluon field

$$
A_{\varphi}^{(1)}=-\frac{\nu_{1}}{g_{\mathrm{c}} r}[1-h(r)]\left(\begin{array}{ccc}
-\frac{2}{3} & 0 & 0 \\
0 & \frac{1}{3} & 0 \\
0 & 0 & \frac{1}{3}
\end{array}\right),
$$

where $g_{\mathrm{c}}$ is the QCD coupling and the boundary conditions are

$$
\begin{aligned}
& f \rightarrow 0, \quad g^{\prime} \rightarrow 0, \quad h \rightarrow 1 \quad \text { as } r \rightarrow 0, \\
& f \rightarrow 1, \quad g \rightarrow 1, \quad h \rightarrow 0 \quad \text { as } r \rightarrow \infty .
\end{aligned}
$$

Single valuedness of the condensate requires that $\nu_{1}$ be an integer. Antigreen-antidown $(\bar{g} \bar{d})$ and antiblue-antistrange $(\bar{b} \bar{s})$ versions, $\Phi^{(2)}$ with $\nu_{2}$ and $\Phi^{(3)}$ with $\nu_{3}$, can be obtained by permuting the diagonal elements.

To obtain the superfluid velocity and angular momentum per baryon of the non-Abelian vortex, we rewrite Eq. (24) as

$$
\Phi^{(1)}=\Delta_{\mathrm{CFL}} e^{\frac{i}{3} \nu_{1} \varphi}\left(\begin{array}{ccc}
e^{\frac{2 i}{3} \nu_{1} \varphi} f(r) & 0 & 0 \\
0 & e^{-\frac{i}{3} \nu_{1} \varphi} g(r) & 0 \\
0 & 0 & e^{-\frac{i}{3} \nu_{1} \varphi} g(r)
\end{array}\right) .
$$

In this form the overall factor of $e^{\frac{i}{3} \nu_{1} \varphi}$ is the $U(1)_{\mathrm{B}}$ phase, while the phase factors within the matrix are a color rotation. [We note for later computation of the covariant derivative of $\Phi^{(1)}$ that the gradients of these phases are compensated by the color gauge field (25).]

The chemical potential per quark pair is $2 \mu_{\mathrm{q}}=\frac{2}{3} \mu_{\mathrm{B}}$, so from Eqs. (6), (7), and (9) the total momentum per quark pair is related to the superfluid velocity $\vec{v}$ by

$$
\vec{p}=\frac{1}{3} \cdot \frac{\nu_{1}}{r} \hat{\varphi}=\frac{2}{3} \mu_{\mathrm{B}} \vec{v}
$$

The circulation around the vortex, Eq. (12), is

$$
C_{(1)}=\oint_{\mathcal{C}} \vec{v} \cdot d \vec{\ell}=\frac{\pi \nu_{1}}{\mu_{\mathrm{B}}} .
$$

Correspondingly, the angular momentum per baryon of the vortex of the form (24) or (27) is

$$
\frac{L_{(1) z}}{N_{\mathrm{B}}}=\frac{1}{2} \nu_{1} .
$$

The same relations also hold for $\Phi^{(2)}$ with $\nu_{2}$ and $\Phi^{(3)}$ with $\nu_{3}$.

We see from Eqs. (1) and (29) and from Eqs. (15) and (30) that singly quantized $\left(\nu_{\mathrm{B}}=1\right)$ vortices in hadronic matter can match onto singly quantized $\left(\nu_{1}=1, \nu_{2}=1\right.$, or $\left.\nu_{3}=1\right)$ non-Abelian vortices in CFL quark matter at a crossover between these phases, with no discontinuity in baryon velocity and angular momentum.

This result can be understood intuitively as follows. In the hadronic vortex, the dibaryon condensate acquires a phase of $2 \pi$ as one follows it along a contour encircling the vortex core. Since the dibaryon can be viewed as three diquarks, this corresponds to each diquark acquiring a phase of $2 \pi / 3$. The non-Abelian vortex in the CFL condensate has exactly the same circulation: each diquark acquires a phase ${ }^{4}$ of $2 \pi / 3$.

We conclude, in agreement with Ref. [11], that a single non-Abelian CFL vortex has the same circulation as a hadronic vortex. However, Ref. [11] suggests that, in order to neutralize the color flux contained in the non-Abelian vortices, three non-Abelian CFL vortices must merge to form a boojum at the CFL-hadronic boundary to which three hadronic vortices then connect [see Fig. 1(b)]. As we argue below, there is no need for such a boojum: a single non-Abelian CFL vortex can smoothly evolve into a single hadronic vortex [as in Fig. 1(c)]. To show this, further consideration of the flavor structure of the vortices is necessary in the hadronic and the CFL phases, as we discuss in Sec. III D.

\section{Gauge-invariant description}

In Sec. III we described the CFL condensate in the unitary gauge. Although such a gauge-fixed description is convenient for writing down the non-Abelian vortex solution explicitly and showing the continuity of the circulation and angular momentum between the hadronic

\footnotetext{
${ }^{4}$ If $U(1)_{\mathrm{B}}$ were a local gauge symmetry, the vortex would become a $U(1)_{\mathrm{B}}$ flux tube. The hadronic vortex and the nonAbelian vortex would both have the same $U(1)_{\mathrm{B}}$ flux in their cores.
} 
phase and the CFL phase, it is not clear how the flavor structures in the two phases are connected. To resolve this problem, in this section we describe vortices in the CFL phase in a gauge-invariant manner [28] using diquarks in Eqs. (16) and (17) as building blocks. We can write down mesonlike and baryonlike gauge-invariant combinations of diquark operators,

$$
\begin{gathered}
\hat{\mathcal{M}}_{i}^{j}(\vec{r}) \equiv \hat{\Phi}_{i \alpha}^{\dagger} \hat{\Phi}^{\alpha j}, \\
\hat{\Upsilon}^{i j k}(\vec{r}) \equiv \frac{1}{6} \epsilon_{\alpha \beta \gamma} \hat{\Phi}^{\alpha i} \hat{\Phi}^{\beta j} \hat{\Phi}^{\gamma k} .
\end{gathered}
$$

We focus on $\hat{\Upsilon}^{i j k}(\vec{r})$ for the moment and consider $\hat{\mathcal{M}}_{i}^{j}(\vec{r})$ later in Sec. III D 3. According to quark-hadron continuity, $\left\langle\hat{\Upsilon}^{i j k}(\vec{r})\right\rangle$ is nonzero in both the CFL and hadronic phases because both phases break baryon number, via diquark and dibaryon condensates, respectively. In Secs. III D 1 and III D 2 below we discuss the projection of $\hat{\Upsilon}^{i j k}(\vec{r})$ onto specific flavor representations.

In the CFL phase, in the mean-field approximation,

$$
\Upsilon^{i j k}(\vec{r}) \equiv\left\langle\hat{\Upsilon}^{i j k}(\vec{r})\right\rangle=\frac{1}{6} \epsilon_{\alpha \beta \gamma} \Phi^{\alpha i} \Phi^{\beta j} \Phi^{\gamma k}
$$

$\Upsilon^{i j k}(\vec{r})$ provides a gauge-invariant description of the nonAbelian vortex originally defined through the gaugedependent condensate $\Phi$.

Note that the irreducible flavor $S U(3)$ decomposition of $\Upsilon^{i j k}(\vec{r})$ is

$$
3^{*} \otimes 3^{*} \otimes 3^{*}=1 \oplus 8 \oplus 8 \oplus 10^{*},
$$

so that not only flavor-singlet but also flavored vortices can be obtained from $\Phi$ by appropriate projections. These would match to certain of the hadronic vortices classified in Eq. (14).

According to (33) the total number of 6-quark condensates in the CFL phase is $3 \times 3 \times 3=27$, while the number of pairs of octet baryons in the hadronic phase is $8 \times 8=64$. One might think that there is a mismatch, but this is because our diquark condensate $\Phi$ only includes flavor antisymmetric diquarks. We discuss this point in Sec. III D 2.

In the hadronic phase a nonzero expectation value of $\hat{\Upsilon}^{i j k}(\vec{r})$ is an order parameter for baryon number violation, which is manifest with $\hat{\Upsilon}^{i j k}(\vec{r})$ rewritten in terms of the baryon-interpolating operator, $\hat{B}_{j}^{i a} \equiv \hat{\Psi}^{\alpha i} \hat{q}_{\alpha j}^{a}$; the spin $1 / 2$ is represented by the index $a$ on $q_{\alpha j}^{a}$. In writing $\hat{B}_{j}^{i a}$ as interpolating operators for spin- $1 / 2$ baryons, we simplify the operator structure by neglecting the axial vector diquark (called the "bad diquark" in hadron structures), which is a reasonable approximation for low-lying baryons. The operator $\hat{B}_{m}^{i a}$ can be written as a sum of flavor-singlet and flavor-octet operators as

$$
\hat{B}_{m}^{i a}=\hat{B}_{\mathbf{1}}^{a}\left(\delta_{m}^{i} / \sqrt{6}\right)+\hat{B}_{\mathbf{8}}^{A a}\left(t^{A}\right)_{m}^{i},
$$

where the $t^{A}$ are the $\mathrm{SU}(3)$ generators $(A=1, \ldots, 8)$ in flavor space, with the normalization $\operatorname{tr}\left(t^{A}\right)^{2}=1 / 2$. Then $\hat{B}_{1}^{a} \equiv 2 \operatorname{tr}\left(\hat{B}^{a}\right) / \sqrt{6}$ and $\hat{B}_{\mathbf{8}}^{A a} \equiv 2 \operatorname{tr}\left(t^{A} \hat{B}^{a}\right)$.

Forming $\hat{B}_{j}^{i a}$ by combining the quark operator with the diquark operator written in terms of two quarks, (16), we find the operator relation

$$
\hat{\Upsilon}^{i j k}(\vec{r})=\frac{1}{3} \epsilon^{k m n}\left(C \gamma_{5}\right)_{a b} \hat{B}_{m}^{i a} \hat{B}_{n}^{j b}
$$

Clearly, a dibaryon condensate $\langle\hat{B} \hat{B}\rangle \neq 0$ in the hadronic phase makes $\Upsilon^{i j k}$ nonzero.

\section{Flavor-singlet vortex}

We first consider vortices in the flavor-singlet projection of the gauge-invariant order parameter,

$$
\hat{\Upsilon}_{\mathbf{1}}(\vec{r})=\epsilon_{i j k} \hat{\Upsilon}^{i j k}(\vec{r})
$$

We can equivalently express this expectation value using Eq. (36) in terms of the baryon operators, (35),

$$
\begin{aligned}
\Upsilon_{\mathbf{1}}(\vec{r}) & =\frac{1}{3}\left(C \gamma_{5}\right)_{a b}\left(\delta_{i}^{m} \delta_{j}^{n}-\delta_{i}^{n} \delta_{j}^{m}\right)\left\langle\hat{B}_{m}^{i a} \hat{B}_{n}^{j b}\right\rangle \\
& =\frac{1}{3}\left(C \gamma_{5}\right)_{a b}\left(\left\langle\hat{B}_{\mathbf{1}}^{a} \hat{B}_{\mathbf{1}}^{b}\right\rangle-\frac{1}{2}\left\langle\hat{B}_{\mathbf{8}}^{A a} \hat{B}_{\mathbf{8}}^{A b}\right\rangle\right) ;
\end{aligned}
$$

in hadronic language $\Upsilon_{1}(\vec{r})$ corresponds to a flavor-singlet condensate made with flavor-singlet and flavor-octet baryons.

In the CFL phase insertion of any of $\Phi^{(1)}, \Phi^{(2)}$ or $\Phi^{(3)}$ gives the same form

$$
\Upsilon_{\mathbf{1}}=e^{i \nu_{q} \varphi} \Delta_{\mathrm{CFL}}^{3} f(r) g^{2}(r),
$$

which implies that the non-Abelian vortices $\Phi^{(1,2,3)}$ have a common flavor-singlet component. A singly quantized $\left(\nu_{q}=1\right)$ vortex has the same circulation $2 \pi / 2 \mu_{B}$ as a singly quantized $\left(\nu_{\mathrm{B}}=1\right)$ hadronic vortex in the flavorsinglet channel; its phase winds by $2 \pi$ on a contour encircling the vortex core, consistent with our finding that these two vortices match smoothly onto each other, with quantized vortex circulation $2 \pi / 2 \mu_{B}$.

If, on the other hand, we were to substitute the field configuration for an Abelian vortex $\Phi^{(\mathrm{A})}$ in Eq. (19) into Eq. (37), we would find 


$$
\Upsilon_{\mathrm{A}}=e^{3 i \nu_{\mathrm{A}} \varphi} \Delta_{\mathrm{CFL}}^{3} f^{3}(r) ;
$$

the gauge-invariant form of a singly quantized Abelian vortex winds three times more (by $6 \pi$ ) on a contour encircling the vortex core. This winding is consistent with needing three hadronic vortices to match to one Abelian vortex [11].

We now consider the vortex energy in terms of the gauge-invariant order parameter. Because of the boundary condition (26), the extra energy density of a vortex far away from its core arises from the derivative terms; for a nonAbelian vortex the energy density is asymptotically

$$
\epsilon^{(1)}=\operatorname{tr}\left|\boldsymbol{D} \Phi^{(1)}\right|^{2},
$$

where the covariant derivative is $\boldsymbol{D}=\boldsymbol{\nabla}-i g_{\mathrm{c}} \mathbf{A}$, and the trace is taken with respect to color-flavor matrix indices. The gluon field (25) in $\boldsymbol{D}$ exactly cancels the derivatives of the phases in the color-flavor matrix part of $\Phi_{\alpha i}^{(1)}$ in Eq. (27). As a result only the derivative of the $U(1)_{\mathrm{B}}$ phase contributes to the energy density at large distance from the vortex core,

$$
\epsilon^{(1)}=3 \cdot \frac{\nu_{1}^{2}}{9 r^{2}}\left|\Delta_{\mathrm{CFL}}\right|^{2} .
$$

Calculating $\nabla \Upsilon_{1}$ from Eq. (39) we can write the energy in terms of the gauge-invariant order parameter as

$$
\epsilon_{1}=\frac{1}{3\left(\Delta_{\mathrm{CFL}}\right)^{4}}\left|\nabla \Upsilon_{1}\right|^{2}
$$

This is the kinetic term of a Ginzburg-Landau theory [29] at large distance for the gauge-invariant flavor-singlet order parameter $\Upsilon_{\mathbf{1}}$.

We can write the full gauge-invariant Ginzburg-Landau free energy in two dimensions in the form

$$
F=\mathcal{N} \int d^{2} r\left(\left|\boldsymbol{\nabla} \tilde{\Upsilon}_{\mathbf{1}}\right|^{2}-m^{2}\left|\tilde{\Upsilon}_{\mathbf{1}}\right|^{2}+\frac{\lambda}{2}\left|\tilde{\Upsilon}_{\mathbf{1}}\right|^{4}\right)
$$

where we rescale $\Upsilon_{1} \rightarrow \tilde{\Upsilon}_{1}$ to make the coefficient of the gradient term be unity at the mean-field level. The full determination of the coefficients, $m^{2}$ and $\lambda$, from QCD is a challenging future problem. This form of the GinzburgLandau free energy describes the interaction between the flavor-singlet parts of non-Abelian vortices (see also Ref. [30]).

As in simple superfluids, e.g., ${ }^{4} \mathrm{He}$, the interaction energy of two non-Abelian vortices in the gauge-invariant picture is essentially the integral of the product of the two vortex velocities, $v_{1} \cdot v_{2}$, which is generally negative between two similarly quantized vortices; for two singly quantized vortices whose cores are separated by $L$, assumed much greater than the coherence length $1 / m$, the interaction energy is ${ }^{5}$

$$
F_{\text {int }}=-\frac{2 \pi m^{2}}{\lambda} \ln (m L) .
$$

Here, the coefficient appears from the normalized condensate, $\left|\tilde{\Upsilon}_{\mathbf{1}}\right|^{2}=m^{2} / \lambda$ in the mean-field approximation. This logarithmically diverging result (see [31]) indicates that the two vortices repel.

\section{Flavored vortices}

We now consider vortices in the flavor-octet projection of the gauge-invariant order parameter,

$$
\hat{\Upsilon}_{\mathbf{8}}^{A}=\epsilon_{i j \ell}\left(t^{A}\right)_{k}^{\ell} \hat{\Upsilon}^{i j k}(\vec{r}) .
$$

This term vanishes in the mean-field approximation, but beyond mean field the flavor-octet part of non-Abelian CFL vortices could smoothly connect to flavor-octet hadronic vortices, just as the flavor-singlet part of a non-Abelian vortex can smoothly connect to a flavor-singlet hadronic vortex. As with the flavor singlet, we can express $\Upsilon_{\mathbf{8}}^{a}(\vec{r})$ in terms of the baryon operators,

$$
\begin{aligned}
\Upsilon_{\mathbf{8}}^{A}(\vec{r}) & =\frac{1}{3}\left(C \gamma_{5}\right)_{a b} \epsilon_{i j \ell}\left(t^{A}\right)_{k}^{\ell} \epsilon^{k m n}\left\langle\hat{B}_{m}^{i a} \hat{B}_{n}^{j b}\right\rangle \\
& =\frac{1}{6}\left(C \gamma_{5}\right)_{a b}\left(d^{A B C}\left\langle\hat{B}_{\mathbf{8}}^{B a} \hat{B}_{\mathbf{8}}^{C b}\right\rangle-\frac{\sqrt{6}}{3}\left\langle\hat{B}_{\mathbf{1}}^{a} \hat{B}_{\mathbf{8}}^{b}\right\rangle\right),
\end{aligned}
$$

where the $d$ tensor is defined by $\left\{\lambda^{A}, \lambda^{B}\right\}=$ $\frac{1}{3} \delta^{A B}+d^{A B C} \lambda^{C}$. Equation (47) shows how the flavor-octet vortex $\Upsilon_{\mathbf{8}}^{A}$ can be understood as a symmetric 8 made with two octet baryons [as classified in Eq. (14)].

We note that the flavor structure of dibaryon pairings such as $\langle n n\rangle$ and $\langle p p\rangle$ in two-flavor superfluid nuclear matter cannot be realized in the present setup for the CFL phase. For example, a neutron pair condensate, $\langle n n\rangle$, has an overlap with the diquark condensate, $\langle u d\rangle\langle u d\rangle\langle d d\rangle$; however, because $\langle d d\rangle$ is flavor symmetric, it must be color symmetric for a spin-singlet (antisymmetric) pair, and thus cannot be constructed out of $\Upsilon^{i j k}$ given in terms of $\Phi^{\alpha i}$. Such pairing is possible in the color sextet channel; although single gluon exchange is repulsive for colortriplet diquarks, and such pairing is presumably less

\footnotetext{
${ }^{5}$ The interaction free energy of two vortices, one at the origin with phase $\phi_{1}$ and the second with phase $\phi_{2}$, where the $\phi$ 's are the azimuthal angles $\varphi$ measured from the individual vortex cores, is $F_{\text {int }}=\int d^{2} r \boldsymbol{\nabla} \phi_{1} \cdot \nabla \phi_{2}\left|\tilde{\Upsilon}_{0}\right|^{2}$. After integration by parts only the surface term remains, since $\nabla^{2} \varphi=0$, and choosing the branch cut in the phase along the $x$ axis, the integral becomes $\int_{1 / m}^{L} d x \partial_{y} \phi_{1} \cdot \Delta \phi_{2}\left|\tilde{\Upsilon}_{0}\right|^{2}$. Since the discontinuity of $\varphi_{2}$ across the $x$ axis, $\Delta \phi_{2}$, is $-2 \pi$ (except at its core, where the order parameter vanishes), we find Eq. (45).
} 
favored, this pairing breaks the same symmetries and is therefore induced by color antisymmetric pairing $[3,32]$. Another possible way to form $\langle d d\rangle$ is with color-triplet and spin-triplet pairing [33,34], which has spin 1 and breaks rotational symmetry. Such states could connect naturally to ${ }^{3} P_{2}$ pairing in dense nuclear matter. We leave the question of vortex continuity between neutron ${ }^{3} P_{2}$ pairing and colortriplet. spin-triplet paired quark matter for the future.

\section{Flavor symmetry breaking in the vortex core}

At least at the level of the mean-field approximation, flavor symmetry is spontaneously broken in the core of a CFL vortex [12], $S U(3) \rightarrow S U(2) \otimes U(1)$, which can be characterized by the flavor-octet order parameter $\mathcal{M}_{i}^{j}=$ $\left\langle\hat{\mathcal{M}}_{i}^{j}\right\rangle$ introduced in Eq. (31). For a $\Phi^{(3)}$ condensate, for example, we have

$$
\operatorname{tr}\left(t^{A} \mathcal{M}\right)=-\frac{2}{\sqrt{3}}\left[f(r)^{2}-g(r)^{2}\right] \delta^{A, 8} .
$$

Whether this prediction survives beyond mean field requires analysis of the fluctuation modes of a CFL vortex in $(3+1)$ dimensions. If the core is effectively a $(1+1)$ dimensional system, the Mermin-Wagner-HohenbergColeman theorem [35-37] would imply that fluctuations in the order parameter along the symmetry broken directions (the $\mathrm{CP}^{2}$ mode [12]) would prevent spontaneous breaking of continuous symmetries in systems in $(1+1)$ dimensions at $T \geq 0$ [and in $(2+1)$ dimensions at $T>0$ [38]]. This indicates that if the Hamiltonian is flavor symmetric no flavor-breaking condensate would be able to appear in the vortex core. (See Ref. [39] and references therein for detailed discussions on the absence of flavor symmetry breaking in the vortex core in relativistic theories as well as possible exceptions in nonrelativistic theories.) Even if flavor symmetry is spontaneously broken in the CFL vortex, e.g., due to a coupling between the Kelvin mode and the $\mathrm{CP}^{2}$ mode, which requires $(3+1)$-dimensional analysis, the octet components of $\hat{\Upsilon}^{i j k}$ could develop an expectation value inside the hadronic vortex core.

Therefore, in either scenario, the flavor transformation properties of the CFL vortices do not prevent continuity of vortices between the hadronic and CFL phases.

\section{COLOR FLUX}

In Sec. III we argued that at a crossover between the hadronic phase and the CFL phase, a hadronic vortex can smoothly evolve into a non-Abelian CFL vortex, in keeping with quark-hadron continuity. More generally, even if there is a first order phase transition between the CFL and hadronic phase (terminating a CFL vortex in much the same way as vortex terminates at a free surface in a liquid), it is hard to avoid a hadronic vortex, since then one would have to have a layer of discontinuity in the baryonic current. This raises the question of what happens to the color magnetic flux in the non-Abelian CFL vortex. Reference [11] argued that at the quark-hadronic boundary there must be a boojum where three non-Abelian CFL vortices with different color magnetic fluxes come together so that their color fluxes cancel, and they can then connect to three hadronic vortices [see Fig. 1(b)]. However, we argue that there is no need for such an elaborate construction.

The gauge-invariant characterization of the color magnetic flux was recently discussed in Ref. [40], which noted that, just as for local non-Abelian flux tubes [41,42], the color magnetic flux in the non-Abelian CFL vortex can be characterized by the Aharonov-Bohm phase acquired by a heavy "probe" quark when transported around the vortex. This is manifest in the expectation value of the trace of the Wilson loop operator,

$$
W_{3}(\mathcal{C})=\frac{1}{N_{\mathrm{c}}} \operatorname{tr} \mathcal{P} \exp \left(i g_{\mathrm{c}} \oint_{\mathcal{C}} d s_{\mu} A^{\mu A} t_{3}^{A}\right),
$$

where $N_{\mathrm{c}}$ is the number of colors (i.e., $N_{\mathrm{c}}=3$ ), $\mathcal{P}$ denotes path ordering, the $t_{3}^{A}$ are the $S U(3)$ color generators in the triplet representation, and $\mathcal{C}$ is a closed contour encircling the vortex. If the contour is large enough then the Wilson loop follows a perimeter law $\left\langle W_{3}(\mathcal{C})\right\rangle=\chi_{\mathcal{C}} \exp (-\kappa L(\mathcal{C}))$ in both phases, where $L(\mathcal{C})$ is the length of the contour, and $\kappa$ is an effective mass. The prefactor $\chi_{\mathcal{C}}$ contains the Aharonov-Bohm phase for the path $\mathcal{C}$, normalized so that for a large contour $\mathcal{C}_{0}$ that does not encircle a vortex, $\chi_{\mathcal{C}_{0}}=1$.

Reference [40] emphasized that, for a non-Abelian CFL vortex, $\chi_{\mathcal{C}}$ is a $Z_{3}$ phase, an element of the center of the color gauge group, whereas in the hadronic phase we expect that for a contour $\mathcal{C}$ encircling a hadronic vortex there will be no such phase, $\chi_{\mathcal{C}}=1$, since there is no color flux in the hadronic vortex. However, as we now explain, this does not mean that a boojum is required at the quarkhadron boundary.

One of the leading scenarios for explaining confinement is the condensation of "center vortices" [43-47]; for a recent review see [48]. According to this picture, the confining QCD vacuum is filled with flux tubes that carry $Z_{3}$ color flux. It is therefore quite possible that when a nonAbelian CFL vortex arrives at the CFL-hadronic boundary, its color flux can leak away into the confined hadronic phase, indistinguishable from the preexisting condensate of center vortices. There is no reason why multiple CFL vortices should be constrained to converge at a boojum before entering the hadronic phase: even if they are far apart their color fluxes can still cancel by connecting with each other through the putative condensate of color vortices in the hadronic phase.

Now, let us consider a spherical compact stellar object made of $S U(3)$-symmetric matter, rotating so slowly around the central $z$ axis that it contains exactly one vortex 
lying along this axis. We assume that the lower-density mantle is in the hadronic phase and the higher-density core is in the CFL phase. The vortex has a "southern" hadronic segment, a central CFL segment, and a "northern" hadronic segment, Since such a system cannot contain a boojum, which requires three vortices, what then is configuration of the color flux? When the CFL vortex reaches the hadronic phase, at the north pole of the core, its $U(1)_{\mathrm{B}}$ circulation becomes the northern segment of the hadronic vortex, which continues upwards along the $z$ axis.

In the center vortex picture, its color flux would become another member of the existing condensate of center vortices in the hadronic phase. That color flux is redistributed through a chain of monopoles and antimonopoles connected by color flux tubes [48] in the hadronic mantle and ultimately links to the south pole of the core, where it would combine with the southern segment of the hadronic vortex to create the CFL vortex that begins at the south pole of the core.

\section{CONCLUSIONS}

We have argued here that singly quantized superfluid vortices in three-flavor symmetric hadronic matter can transform smoothly into singly quantized non-Abelian superfluid vortices in three-flavor symmetric color-flavor locked quark matter, without the need to include boojums to mark the transition at the interface between the two phases. One can make a one-to-one correspondence between vortices in the baryonic and quark phases. We have constructed a gauge-invariant description of nonAbelian vortices. A natural next step is to spell out the full Ginzburg-Landau theory for non-Abelian vortices in terms of their gauge-invariant order parameter.

We have only studied the question of the connections of single vortices in fully $S U(3)$ flavor symmetric matter. To make our analysis applicable to more realistic situations in neutron stars where one does not have even isospin symmetry requires extending the analysis to flavor-symmetry broken states, resulting from the higher mass of the strange quark (for a discussion of the ramifications for CFL superfluid vortices see Ref. [12]). The extension requires considering BCS pairing states in the quark phase beyond ideal CFL with simple color, flavor, and spin asymmetry. Ultimately we would like to determine the extent to which one can connect the hadronic and quark matter phases and their vortices in a smooth way. Furthermore, at large rotational rates one expects an array of vortices. While in the hadronic phase the vortices are expected to form a triangular lattice; determining the optimal lattice configurations in the quark phase requires better understanding the interactions of non-Abelian vortices. For such systems with the interactions of multiple vortices, other types of connections from multiple non-Abelian vortices to multiple hadronic vortices may be realized by dynamics, which is not excluded from our analysis on single vortex.

\section{ACKNOWLEDGMENTS}

The authors thank Aleksey Cherman, Muneto Nitta, and Srimoyee Sen for useful discussions. Research of author G. B. was supported in part by NSF Grants No. PHY1305891 and No. PHY1714042. Author T. H. was supported by JSPS Grants-in-Aid for Scientific Research No. $15 \mathrm{H} 03663$ and No. 18H05236; G. B. and T. H. were partially supported by the RIKEN iTHES Project and iTHEMS Program. M. G. A. is supported by the U.S. Department of Energy, Office of Science, Office of Nuclear Physics under Award No. DE-FG02-05ER41375. K. F. was supported by Japan Society for the Promotion of Science (JSPS) KAKENHI Grants No. 15H03652, No. 15K13479, and No. 18H01211. M. T. was supported by JSPS Grant-inAid for Scientific Research, Grant No. 16K05357. The authors are grateful to the Aspen Center for Physics, supported by NSF Grant No. PHY1607611, where part of this research was done.
[1] M. G. Alford, K. Rajagopal, and F. Wilczek, Nucl. Phys. B537, 443 (1999).

[2] T. Schäfer and F. Wilczek, Phys. Rev. Lett. 82, 3956 (1999).

[3] M. G. Alford, J. Berges, and K. Rajagopal, Nucl. Phys. B558, 219 (1999).

[4] M. M. Forbes and A. R. Zhitnitsky, Phys. Rev. D 65, 085009 (2002).

[5] K. Iida and G. Baym, Phys. Rev. D 66, 014015 (2002).

[6] K. Iida and G. Baym, Phys. Rev. D 65, 014022 (2001).

[7] N. D. Mermin, Surface singularities and superflow in 3He-A, in Quantum Fluids and Solids, edited by S. B. Trickey, E. D. Adams, and J. W. Dufty (Plenum, New York, 1977), pp. 3-22.
[8] A. P. Balachandran, S. Digal, and T. Matsuura, Phys. Rev. D 73, 074009 (2006).

[9] E. Nakano, M. Nitta, and T. Matsuura, Phys. Rev. D 78, 045002 (2008).

[10] M. G. Alford, S. K. Mallavarapu, T. Vachaspati, and A. Windisch, Phys. Rev. C 93, 045801 (2016).

[11] M. Cipriani, W. Vinci, and M. Nitta, Phys. Rev. D 86, 121704 (2012).

[12] M. Eto, Y. Hirono, M. Nitta, and S. Yasui, Prog. Theor. Exp. Phys. (2014) 12D01.

[13] D. K. Hong and S. D. H. Hsu, Phys. Rev. D 68, 034011 (2003). 
[14] M. G. Alford, A. Schmitt, K. Rajagopal, and T. Schäfer, Rev. Mod. Phys. 80, 1455 (2008).

[15] D. T. Son and M. A. Stephanov, Phys. Rev. D 61, 074012 (2000).

[16] D. T. Son and M. A. Stephanov, Phys. Rev. D 62, 059902 (2000).

[17] M. Rho, A. Wirzba, and I. Zahed, Phys. Lett. B 473, 126 (2000).

[18] K. Fukushima, Phys. Rev. D 70, 094014 (2004).

[19] T. Hatsuda, M. Tachibana, and N. Yamamoto, Phys. Rev. D 78, 011501 (2008).

[20] E. H. Fradkin and S. H. Shenker, Phys. Rev. D 19, 3682 (1979).

[21] T. Hatsuda, M. Tachibana, N. Yamamoto, and G. Baym, Phys. Rev. Lett. 97, 122001 (2006).

[22] H. Abuki, G. Baym, T. Hatsuda, and N. Yamamoto, Phys. Rev. D 81, 125010 (2010).

[23] K. Fukushima and T. Hatsuda, Rep. Prog. Phys. 74, 014001 (2011).

[24] G. Baym, T. Hatsuda, T. Kojo, P. D. Powell, Y. Song, and T. Takatsuka, arXiv:1707.04966.

[25] G. Baym, The microscopic description of superfluidity, in Mathematical Methods in Solid State and Superfluid Theory: Scottish Universities' Summer School, edited by R. C. Clark and G. H. Derrick (Springer, Boston, MA, 1968), pp. 121-156.

[26] T. Inoue, S. Aoki, T. Doi, T. Hatsuda, Y. Ikeda, N. Ishii, K. Murano, H. Nemura, and K. Sasaki (HAL QCD Collaboration), Nucl. Phys. A881, 28 (2012).

[27] T. Inoue, N. Ishii, S. Aoki, T. Doi, T. Hatsuda, Y. Ikeda, K. Murano, H. Nemura, and K. Sasaki (HAL QCD Collaboration), Prog. Theor. Phys. 124, 591 (2010).

[28] K. Rajagopal and F. Wilczek, At the frontier of particle physics, in Handbook of $Q C D$, edited by M. Shifman and
B. Ioffe (World Scientific, Singapore, 2001), Vol. 1-3, pp. 2061-2151.

[29] K. Iida and G. Baym, Phys. Rev. D 63, 074018 (2001); 66, 059903(E) (2002).

[30] R. Auzzi, M. Eto, and W. Vinci, J. High Energy Phys. 02 (2008) 100.

[31] I. Khalatnikov, An Introduction to the Theory of Superfluidity (W. A. Benjamin, New York, 1966).

[32] R. D. Pisarski and D. H. Rischke, in A Symposium in Memory of Judah M. Eisenberg: Nuclear Matter, Hot and Cold Tel Aviv, Israel, 1999 (Tel Aviv University, Tel Aviv, 2000).

[33] M. G. Alford, J. A. Bowers, J. M. Cheyne, and G. A. Cowan, Phys. Rev. D 67, 054018 (2003).

[34] A. Schmitt, Phys. Rev. D 71, 054016 (2005).

[35] N. D. Mermin and H. Wagner, Phys. Rev. Lett. 17, 1133 (1966).

[36] P. C. Hohenberg, Phys. Rev. 158, 383 (1967).

[37] S. R. Coleman, Commun. Math. Phys. 31, 259 (1973).

[38] S.-k. Ma and R. Rajaraman, Phys. Rev. D 11, 1701 (1975).

[39] M. Nitta, S. Uchino, and W. Vinci, J. High Energy Phys. 09 (2014) 098.

[40] A. Cherman, S. Sen, and L. G. Yaffe, arXiv:1808.04827.

[41] J. Preskill and L. M. Krauss, Nucl. Phys. B341, 50 (1990).

[42] M. G. Alford and J. March-Russell, Nucl. Phys. B369, 276 (1992).

[43] G. 't Hooft, Nucl. Phys. B138, 1 (1978).

[44] P. Vinciarelli, Phys. Lett. B 78B, 485 (1978).

[45] T. Yoneya, Nucl. Phys. B144, 195 (1978).

[46] J. M. Cornwall, Nucl. Phys. B157, 392 (1979).

[47] H. B. Nielsen and P. Olesen, Nucl. Phys. B160, 380 (1979).

[48] J. Greensite, EPJ Web Conf. 137, 01009 (2017). 